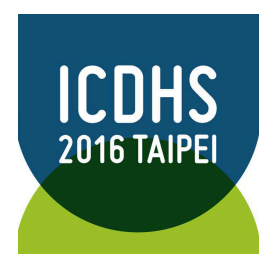

\title{
Why do people check in to emergency room on Facebook: A content analysis of users' check-in posts at National Taiwan University Hospital
}

Tsung-Yeh Vincent Lee / Hong-Shiu Luke Liang / Ming-Hsin Phoebe Chiu / National Taiwan Normal University / Taipei / Taiwan

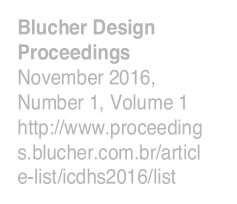

\begin{abstract}
Designing for emergency preparedness with the advantage of social media has become a priority for mobile app developers, medical practitioners, and agencies from public sector. To uncover the possibility of location-based social media use in life-saving emergent situations, this study takes a qualitative content analysis approach to identify the underlying purposes, emotions, and behaviours of the users who check-in the hospital emergency room on social media. A leading medical centre in Taiwan was selected as the data collection site. A total of 1,063 location-based check-in posts from January to May 2015 were collected and analysed.
\end{abstract}

\section{Keywords}

Self-disclosure, location-based service, Social Network Sites, Facebook Check-in Service

\section{Introduction}

The use of social media is not only popular for social and entertainment purposes, but is also prevalent in reporting emergency. A recent American Red Cross survey reported that more people now use social media tools to report emergencies or call for help, to seek and share information or to inform their relatives and friends that they are safe and well. Medical practitioners are strictly confidential about patient information available online because details of the treatment procedures and personal health records and history are personal. In contrast, many users or even patients choose to disclose their health information by themselves on SNSs visibly. Designing for emergency preparedness with the advantage of social media has become a priority for mobile app developers, medical practitioners, and agencies from public sector. To uncover the possibility of location-based social media use in life-saving emergent situations, this study takes a qualitative content analysis approach to identify the underlying purposes, emotions, and behaviors of the users who check-in the hospital emergency room on social media. The Facebook presence of a leading medical center in Taiwan-National Taiwan University Hospital- was selected as the data collection site, and check-in posts at this location from January to May 2015 were collected, resulting in a total of 1,063 location-based check-in posts.

\section{Literature review Self-disclosure on SNSs}

Nowadays, SNSs (Social Networking Sites) have become extremely prevalent with billions of users around the world. People tend to disclose their personal information more than anytime ever as the SNSs grows popular. Almost all SNSs are based on the sharing personal information at a certain level. It allows, and sometimes even, encourages their members to self-disclose and self-present by maintaining their personal profiles and by posting photos, videos, and preferences (Ledbetter et al., 2011). Self-disclosure refers to the act of revealing personal information to another person (Derlega \& Chaikin, 1977). It is also defined as the behavior of revealing private or personal information about oneself to others (Archer and Burleson, 1980) to facilitate the establishment of mutual understanding, intimacy, and trust in interpersonal relationships (Varnali \& Toker, 2015).

An abundance of research effort has been made in regard to self- disclosure. However, as Varnali and Toker (2015) pointed that, even though many researchers have made a great deal of contribution toward the understanding of self-disclosure in computer-mediated communication (CMC), but most of them are conducted in 
experiment settings instead of actual social setting. Lee, Noh, and Koo (2013) also argued that the effects caused by self-disclosure remained not clear.

\section{Location-Based service and Facebook Check-in service}

A location-based service (LBS) is defined as "any service or application that extends spatial information processing, or GIS capabilities, to end users via the Internet and/or wireless network" (Koeppel, 2000). Facebook launched "Places" in August 2010. It is a check-in service, with embedded LBS features, allowing Facebook users to notify their friends to their presence at a particular location. This service discloses one's location, and shares activities with others, and establishes links between his/her physical and social worlds.

The recent rapid expansion of use of mobile devices, such as smart phones and tablets, with global positioning systems (GPS) capabilities has facilitated the massive usage of LBS, such as Facebook Check-in service. Lin et al. (2014) conducted a study about why people continue to use such service. The results indicated that perceived playfulness exerted a significant influence on satisfaction and continuance intention, but privacy concerns did not seem to influence the direct path between satisfaction and continuance intention. However, none of the research efforts on location-based information disclosure has yet answered the question regarding why on social media do people check-in to places where medical attention may be needed.

\section{Social media use in emergency situation}

According to a survey study conducted by American Red Cross in 2012, social media and apps would motivate people to prepare in emergency situation (American Red Cross, 2012). The key findings in this study include: (1) Social users are also most likely to seek and share information during emergencies; (2) 76 percent Americans expect help in less than three hours of posting a request on social media; (3) 40 percent of those surveyed mentioned they would use social media tools to inform others that they are safe.

Because of the increasing social media use in emergencies, many look into the potential of implementing and integrating social media to cope with disaster and emergency preparedness. Sanghoee (2015) highlighted Facebook's new feature called Safety Check, and its ability to help people track their friends and family during the suffering period of Nepal's tragic earthquake in 2015. She also stated that "Emergency response is enhanced tremendously by information, especially about people's whereabouts, and social media is uniquely equipped to provide that to first responders." Merchant, Elmer, and Lurie (2011) argued that social media "can also be used to improve preparedness by linking the public with day-to-day, real-time information about how their community's health care system is functioning." They also suggest the need of the studies to evaluate the reliability and validity of public health-related information communicated through social media.

Identifying why people use social media to check in emergency rooms, and analyzing the content and characteristics of their posts on Facebook may provide fruitful insights on enhancing emergency preparedness and medical resources allocation.

\section{Study design and methods}

In order to uncover the reason why people check in to hospital emergency room on Facebook and what they comment on the such life events, we selected one of the leading national medical center in Taiwan- National Taiwan University Hospital, and collected the Facebook check-in posts at this location from January 1, 2015 to May 19, 2015. A total of 1,063 Facebook check-in posts were collected and analyzed.

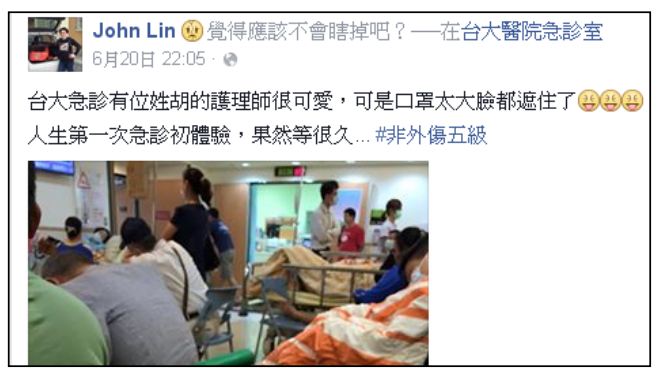

Fig. 1: Example check-in post at NTUH

\section{Data collection and analysis}

National Taiwan University Hospital (NTUH) was founded in 1895. Currently, the hospital has over 6,000 employees, 2,400-odd beds and serves over 8,000 outpatients daily. Over the last hundred plus years since its founding, NTUH and its' medical school has nurtured countless professionals in medicine, including medical students, specialists, pharmacists, nurses and technicians. With its respect to medical service, the quality of NTUH's clinical practice is well known and highly trusted by the people of Taiwan. Therefore, ER of NTUH is one of the most popular check-in locations in hospitals in Taiwan. 
The content of check-in post we collected including text message, emoticon, photos, links and such. An example post is shown in Figure 1.

After collecting the first 271 sets of data to identify the grounding coding schemes, we were able to divide the research themes into three parts: " Format " " Purposes " " Identity Disclosure". We divided these three categories into the following items:

- $\quad$ Format - Check-in only; with text; with selfie; with photo (real time); with photo (other source), with emoticon; with film; with link.

- $\quad$ Purposes - Describing Conditions; Describing Treatment Effect; Expressing Emotions; Complaining; Showing Blessing; Asking Questions; Can not Identified; and Others.

Identity Disclosure - Patient; Patient's Friends/Relatives; Health Care Professionals (Doctor or Nurses); Hospital Volunteers; Non-Emergency Related Person; Can not Identified; and Others.

In order to better understand the purposes, we further divided "Expressing Emotions" into the users' specific observable emotions, including negative moods such as tired, angry, sad, scared, worried, sick, bothered, confused, and positive moods such as pleased and relieved. "Complaining" is an important finding in the research as well, whom and what users complained to were two aspects we attempted to understand.

\section{Results \\ Formats}

Different information is articulated in different formats of posts on Facebook. Emotions are commonly shown on posts with emoticons. Real-time photos may display patient's health conditions, physically or mentally, such as bleeding cut on the body or an angry face.

As shown in Figure 2, "Check-in with text" is the most common form at that people use to checkin at NTUH E.R(53\%), followed by "Check-in with photo (real-time)" (29.8\%), "Check-in with selfie" (11.9\%), "Check-in with emoticon"(11.1\%), "Check-in Only" (8.3\%), and "Check-in with photo (other source)" (3.0\%). The least two are "Check-in with film" and "Check-in with link", which are both fewer than $0.5 \%$. The percentage of "Check-in with selfie" and "Check-in with emoticon" are almost the same.

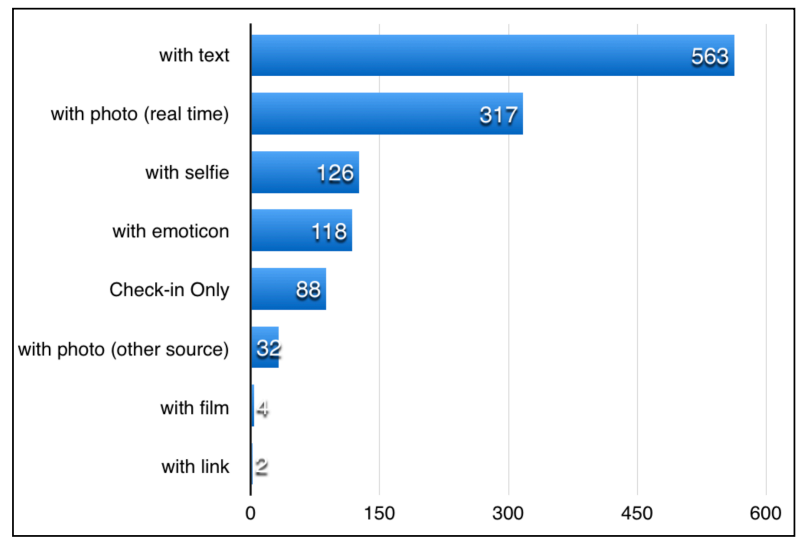

Fig. 2: Formats of check-in posts at NTUH

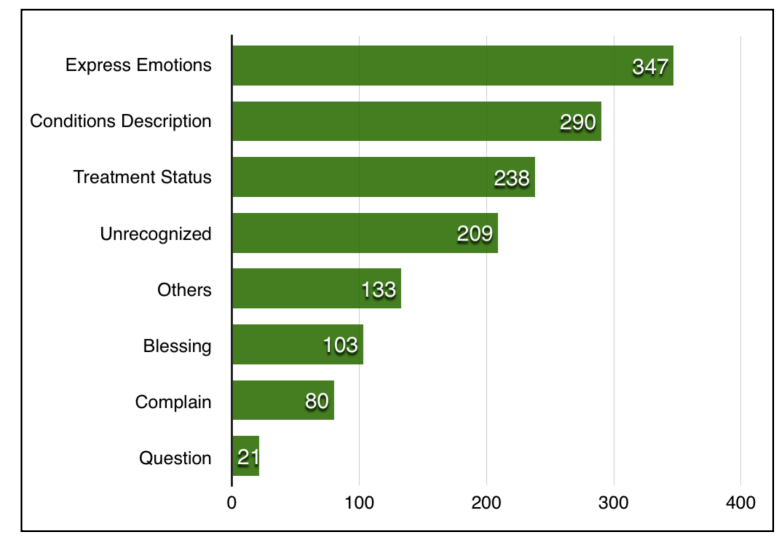

Fig. 3: Purposes of check-in posts at NTUH

\section{Purposes}

As shown in Figure 3, "Expressing Emotions" is the main reason that people check-in E.R on social media (32.6\%). In the category, we found "Sad" (21.49\%), "Worried" (20.92\%) and "Tired" (18.62\%) were the most common negative emotions in those users, as shown in Table 1. We were able to define their emotions by emoticon on posts, the crying face or the sad face appeared in the beginning of the post. Over all, people check-in E.R. were mostly in negative emotions. Surprisingly, some positive emotions also appear in the emergency room, for example, "Pleased" (3.15\%) or "Relieved" (2.58\%), they are found especially when the patient finished their treatment and prepared to leave E.R. "Describing Conditions" (27.3\%) and "Describing Treatment Effect" (22.4\%) are both important purposes.

The users wanted to share situation in real time to friends/relatives on Facebook. Sometimes users asked for further information (9.7\%) about their situation and solicited advices. Religion also plays a role in the purposes, users hope to be blessed by praying or pasting a chapter of Sutra $(9.7 \%)$. There're only $7.5 \%$ users com- 


\begin{tabular}{|l|l|l|}
\hline Emotions & $\begin{array}{l}\text { Check- } \\
\text { in No. }\end{array}$ & $\begin{array}{l}\text { Percent- } \\
\text { age }\end{array}$ \\
\hline Sad & 75 & $21.49 \%$ \\
\hline Worry & 73 & $20.92 \%$ \\
\hline Tired & 65 & $18.62 \%$ \\
\hline Scare & 42 & $12.03 \%$ \\
\hline Angry & 34 & $9.74 \%$ \\
\hline Exclamation & 13 & $3.72 \%$ \\
\hline Pleasure & 11 & $3.15 \%$ \\
\hline Relieved & 9 & $2.58 \%$ \\
\hline Sick & 8 & $2.29 \%$ \\
\hline Bother & 8 & $2.29 \%$ \\
\hline Confuse & 6 & $1.72 \%$ \\
\hline Other & 5 & $1.43 \%$ \\
\hline Total & 349 & $100.00 \%$ \\
\hline
\end{tabular}

plained, mostly about "Problems of medical resources"(29.33\%) of complain) such as in sufficient bed. Users also engage in self-blame (14.67\%) due to their careless behavior that caused to an accident.

\section{Identity disclosure}

As shown in Figure 4, people who check-in most often in E.R. were friends/relatives of patient (43.6\%). Patient self were also love to check-in in E.R.(28.1\%), and there're lot people we can't tell from posts. There're only few Health care (Doctor or Nurses) (5.2\%), Hospital volunteers $(0.4 \%)$, Nonemergency related person (2.7\%) check-in on Facebook.

\section{Conclusions and implications}

\section{People share their feelings by check-in in E.R}

In this study, we are able to conclude that people share their feelings to friends by complaining, seeking to be blessed, or expressing their emotions on Facebook. It could be that people need emotional support when their family members or themselves are ill. They usually can receive comfort from the responses to their check-in posts.

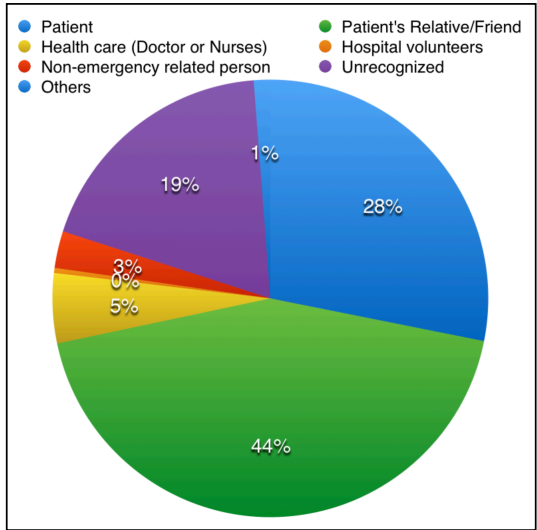

Fig. 4: Identity Disclosure

\section{Real-time information by check-in E.R. may suggest medical resources Allocation}

There are many photos or text posts that describe the status of the check-in posts, like photos with gauze on the wound or arms with drips. They want to inform their friends and relatives of the good news or bad news in E.R. Good news may tamper those who care, some people even selfie their bleeding wound with a smile. On the other hand, knowing the bad situation is effective for offering advices or allocating medical resources with professionals or friends of similar experience.

\section{Most of the expressed emotions are negative}

Since emotion expressing is one of the most common purposes that people check in to E.R. on social media. The expressed emotions were further

divided to 12 sub-categories (Sad, Worried, Tired, Scare...etc.). Only 2 of them are positive, the others are negative. These negative emotions took almost $93 \%$ of all the expressed emotions analyzed in our study. There may be an opportunity for the hospital or healthcare practitioners to identify a better way to release their negative feelings.

\section{Implications of location-based service and SNSs in E.R.}

Our study identified three grounding coding schemes by analyzing 1,063 Facebook check-in posts at NTUH E.R. In the future, it is hoped that more studies on social media use for healthcare setting can be attempted, to show an even greater prospect and dimension of using these results, such as a real time E.R. response system, or automatic E.R. healthcare quality review mechanism, to serve the field of health informatics and social media innovation.

\section{References}

American Red Cross (2012, August 31). More Americans Using Mobile Apps in Emergencies. Retrieved June 25, 2015, from http://www.redcross.org/news/press-release/More-Americans-Using-Mobile-Apps-inEmergencies

Archer, R. L., \& Burleson, J. A. (1980). The Effects of Timing of Self-Disclosure on Attraction and Reciprocity. Journal of Personality and Social Psychology, 38(1), 120-130. doi: 10.1037/0022-3514.38.1.120

Derlega, V. J., \& Chaikin, A. L. (1977). Privacy and Self-Disclosure in Social Relationships. Journal of Social Issues, 33(3), 102-115. doi: 10.1111/j.1540-4560.1977.tb01885.x

Koeppel, I. (2000). What Are Location Services?-From a GIS Perspective. Environmental Systems Research Institute (ERSI) White Paper. 
Ledbetter, A. M., Mazer, J. P., DeGroot, J. M., Meyer, K. R., Yuping M., \& Swafford, B. (2011). Attitudes Toward Online Social Connection and Self-Disclosure as Predictors of Facebook Communication and Relational Closeness. Communication Research, 38(1), 27-53. doi: 10.1177/0093650210365537

Lee, K.T., Noh, M. J., \& Koo, D. M. (2013). Lonely People Are No Longer Lonely on Social Networking Sites: The Mediating Role of Self-Disclosure and Social Support. Cyber Psychology, Behavior \& Social Networking, 16(6), 413-418. doi: 10.1089/cyber.2012.0553

Lin, T.H., Lu, H. P., Hsiao, K. L., \& Hsu, H. H. (2014). Continuance Intention of Facebook Check-in Service Users: An Integrated Model. Social Behavior \& Personality: an international journal, 42(10), 1745-1760. doi: 10.2224/sbp.2014.42.10.1745

Merchant, R. M., Elmer, S., \& Lurie, N. (2011). Integrating Social Media into Emergency-Preparedness Efforts. New England Journal of Medicine, 365(4), 289-291. doi:10.1056/NEJMp1103591

Sanghoee, S. (2015). Facebook: Your emergency rescue of the future? Retrieved June 25, 2015, from http://fortune.com/2015/04/27/facebook-your-emergency-rescue-of-the-future-nepal-earthquake/

Varnali, K., \& Toker, A. (2015). Self-Disclosure on Social Networking. Social Behavior \& Personality: an international journal, 43(1), 1-13. doi: 10.2224/sbp.2015.43.1.1

\section{Biographical Note}

Tsung-Yeh Lee is a PhD student and a librarian of National Kaohsiung Normal University Library. He has worked for an executive search firm from 2008. Then he worked for National Library of Public Information as an Assistant Counselor from 2010. He works for NKNU Library since late 2013.

Hong Shiu Liang is a Ph.D. student in GILIS at NTNU, has worked in at the medical library, university Library as a librarian for 8 years. 2010-2015, he worked in National Library of Public Information as American Corner coordinator. Recently he researches about how to comprehensive high-level reading.

Ming-Hsin Phoebe Chiu is an Associate Professor at the Graduate Institute of Library and Information Studies, National Taiwan Normal University. She currently services as the Executive Editor for Journal of Library and Information Science Research. Her research interests are consumer health informatics, social informatics, and information behavior. 